\title{
Convergence rates in precise asymptotics for a kind of complete moment convergence
}

\author{
Lingtao Kong ${ }^{1}$, Hongshuai Dai ${ }^{1}$
}

\begin{abstract}
In Liu and Lin (Statist. Probab. Letters, 2006), they introduced a kind of complete moment convergence which includes complete convergence as a special case. Inspired by the study of complete convergence, in this paper, we study the convergence rates of the precise asymptotics for this kind of complete moment convergence and get the corresponding convergence rates.
\end{abstract}

Keywords: Convergence rate; precise asymptotics; complete moment convergence MSC(2010): 60F15 60G50

\section{Introduction}

Let $\left\{X, X_{n}, n \in \mathbb{N}\right\}$ be a sequence of i.i.d. random variables. Hsu and Robbins [16] introduced the complete convergence, and proved that if $\mathbb{E}[X]=0$ and $\mathbb{E}\left[X^{2}\right]<\infty$, then

$$
\sum_{n=1}^{\infty} \mathbb{P}\left(\left|S_{n}\right| \geq \epsilon n\right)<\infty, \epsilon>0
$$

where

$$
S_{n}=\sum_{k=1}^{n} X_{k}, n \in \mathbb{N} .
$$

Since then, it has attracted a lot of interest. For example, Erdős [7, 8] proved the necessity. For more information, we refer to Baum and Katz [1], Davis [5, 6], Lai [19] and Gut [9].

Here we point out that the sum in (1.1) tends to infinity as $\epsilon \searrow 0$. Due to this fact, it is interesting to study the precise asymptotic problem, that is, finding an elementary function $f(\epsilon)$ such that $f(\epsilon) \sum_{n=1}^{\infty} \mathbb{P}\left(\left|S_{n}\right| \geq \epsilon n\right)$ has a non-degenerate limit as $\epsilon \searrow 0$. Many results about this topic have been established. For example, Heyde [15] showed that

$$
\lim _{\epsilon \searrow 0} \epsilon^{2} \sum_{n=1}^{\infty} \mathbb{P}\left(\left|S_{n}\right| \geq \epsilon n\right)=\mathbb{E}\left[X^{2}\right],
$$

whenever $\mathbb{E}[X]=0$ and $\mathbb{E}\left[X^{2}\right]<\infty$. For more results on precise asymptotic problems, we refer to Chen [4], Gut and Spătaru [10, 11], Li and Spătaru [20], Spătaru [22, 23] and the references therein.

[1] School of Statistics, Shandong University of Finance and Economics, Jinan 250014, China. E-mail: kltgw277519@126.com (Kong, L.), mathdsh@gmail.com(Dai, H.: Corresponding author). 
Noting the Heyde's result (1.2), it is natural to consider the rate of the convergence for the precise asymptotic problem. This topic has been studied extensively. For example, Klesov [17] proved that if $\mathbb{E}[X]=0, \mathbb{E}\left[X^{2}\right]>0$ and $\mathbb{E}\left[|X|^{3}\right]<\infty$, then

$$
\epsilon^{2} \sum_{n=1}^{\infty} \mathbb{P}\left(\left|S_{n}\right| \geq \epsilon n\right)-\mathbb{E}\left[X^{2}\right]=o\left(\epsilon^{\frac{1}{2}}\right) .
$$

Furthermore, by replacing $\mathbb{E}\left[|X|^{3}\right]<\infty$ in Klesov [17] with $\mathbb{E}\left[|X|^{q}\right]<\infty$ for some $q \in(2,3]$, He and Xie [14 got a much faster rate for Heyde's result. Recently, Gut and Steinebach [12, 13], and Kong [18] also extended the Klesov's [17] result, respectively, and got some new results.

As an extension of the complete convergence, Liu and Lin [21] introduced a new kind of complete moment convergence, and got the precise asymptotic results for this kind of complete moment convergence. These results read as follows.

Theorem 1.1 Let $\left\{X, X_{n}, n \in \mathbb{N}\right\}$ be a sequence of i.i.d. random variables with partial sums $\left\{S_{n}, n \in \mathbb{N}\right\}$. For any $\epsilon>0$, set

$$
\begin{aligned}
& \lambda_{1}(\epsilon, p)=\sum_{n=1}^{\infty} \frac{1}{n^{p}} \mathbb{E}\left[\left|S_{n}\right|^{p} I\left\{\left|S_{n}\right| \geq \epsilon n\right\}\right], \text { for any } p \in[0,2], \\
& \lambda_{2}(\epsilon, \delta)=\sum_{n=2}^{\infty} \frac{(\log n)^{\delta-1}}{n^{2}} \mathbb{E}\left[S_{n}^{2} I\left\{\left|S_{n}\right| \geq \epsilon \sqrt{n \log n}\right\}\right], \text { for any } \delta \in(0,1] .
\end{aligned}
$$

(a) For any $p \in[0,2)$, we have

$$
\lim _{\epsilon \searrow 0} \epsilon^{2-p} \lambda_{1}(\epsilon, p)=\frac{2 \sigma^{2}}{2-p}
$$

if and only if $\mathbb{E}[X]=0$ and $\mathbb{E}\left[X^{2}\right]=\sigma^{2}<\infty$.

(b) For any $\delta \in(0,1]$, we have

$$
\lim _{\epsilon \searrow 0} \epsilon^{2 \delta} \lambda_{2}(\epsilon, \delta)=\frac{\sigma^{2 \delta+2}}{\delta} \mathbb{E}\left[|N|^{2 \delta+2}\right]
$$

if and only if $\mathbb{E}[X]=0, \mathbb{E}\left[X^{2}\right]=\sigma^{2}$ and $\mathbb{E}\left[X^{2}\left(\log ^{+}|X|\right)^{\delta}\right]<\infty$, where $N$ denotes the standard normal random variable and $\log ^{+} x=\log (x \vee e)$.

Similar to the study of the rate of convergence in the complete convergence, it is also interesting to consider the convergence rates in precise asymptotics for Liu and Lin's type complete moment convergence, which is the motivation of our work. In this paper, we will carry out this work.

The rest of this paper is organized as follows. In Section 2, we state the main results of this paper. Section 3 is devoted to presenting the detailed proofs of these results.

We end this section with some notations. We use $C$ to denote a positive constant whose value may vary from place to place. Let $N$ denote the standard normal random variable and $\Phi(x)=\mathbb{P}(|N| \geq x)$ for any $x \geq 0$. 


\section{Main Results}

In this section, we state the main results of this paper. Before we do it, we first introduce some notations. Define

$$
\begin{aligned}
B_{n, \theta} & =\sum_{j=1}^{n} j^{\theta}-\frac{n^{\theta+1}}{\theta+1}, \quad \text { for any }-1<\theta<0 \\
C_{n, \delta} & =\sum_{j=2}^{n} \frac{(\log j)^{\delta-1}}{j}-\frac{(\log n)^{\delta}}{\delta}, \quad \text { for any } 0<\delta \leq 1 .
\end{aligned}
$$

We use $B_{\theta}$ and $C_{\delta}$ to denote the limits of the sequences $\left\{B_{n, \theta}, n \in \mathbb{N}\right\}$ and $\left\{C_{n, \delta}, n \geq 2\right\}$, respectively.

Remark 2.1 Gut and Steinebach [13] proved the convergence of the sequence $\left\{B_{n, \theta} ; n \in\right.$ $\mathbb{N}\}$. For the convergence of the sequence $\left\{C_{n, \delta} ; n \geq 2\right\}$, we will give the proof in Section 3 below.

Now, we state our main results. We have

Theorem 2.1 Let $\left\{X, X_{n}, n \in \mathbb{N}\right\}$ be a sequence of i.i.d. random variables with partial sums $\left\{S_{n}, n \in \mathbb{N}\right\}$. Suppose that

$$
\mathbb{E}[X]=0, \mathbb{E}\left[X^{2}\right]=\sigma^{2}>0, \text { and } \mathbb{E}\left[|X|^{q}\right]<\infty \text { for some } q \in(2,3] .
$$

(a) For any $p \in(0,2)$, we have

$$
\lim _{\epsilon \searrow 0} \epsilon^{(\gamma-1)(2-p)}\left[\epsilon^{2-p} \lambda_{1}(\epsilon, p)-\frac{2 \sigma^{2}}{2-p}\right]=0,
$$

where $\gamma=\frac{q-p}{2 q-2-p}$ and $\lambda_{1}(\epsilon, p)$ is given by (1.4).

(b) For any $0<\delta \leq 1$, we have

$$
\lim _{\epsilon \searrow 0}\left(\log \frac{1}{\epsilon}\right)^{-\delta} \epsilon^{-2 \delta}\left[\epsilon^{2 \delta} \lambda_{2}(\epsilon, \delta)-\frac{\sigma^{2 \delta+2}}{\delta} \mathbb{E}\left[|N|^{2 \delta+2}\right]\right]=0,
$$

where $\lambda_{2}(\epsilon, \delta)$ is defined by (1.5).

Remark 2.2 Under the condition of Theorem 2.1, Theorem 2.1 includes Theorem 1.1 as a special case.

Remark 2.3 It is obvious that $0<\gamma<1$, since $p \in(0,2), q \in(2,3]$.

Remark 2.4 (2.4) obtains the convergence rate for $0<p<2$ in (1.6). When $p=0$, then

$$
\lambda_{1}(\epsilon, 0)=\sum_{n=1}^{\infty} \frac{1}{n^{2}} \mathbb{P}\left(\left|S_{n}\right| \geq \epsilon n\right) .
$$

Under the assumptions of Theorem 2.1, He and Xie [14 got that

$$
\epsilon^{2} \lambda_{1}(\epsilon, 0)-\sigma^{2}=o\left(\epsilon^{q-2}\right) .
$$

Moreover, Liu and Lin [21] also got the precise asymptotics for the case $p=2$. However, the method we used in this paper seems unable to deal with the case $p=2$ and we will study this case in the future work. 
The proof of Theorem 2.1 is based on the following two propositions, one of which is concerned with the Gaussian case (Proposition 2.1) and the other is related to two Berry-Esseen type remainder terms (Proposition 2.2).

Proposition 2.1 Suppose that $\left\{X, X_{n}, n \in \mathbb{N}\right\}$ is a sequence of i.i.d. normal random variables with mean 0 and variance $\sigma^{2}>0$. For any $-1<\theta<0$ and $0<\delta \leq 1, B_{\theta}$ and $C_{\delta}$ denote the limits of the sequences $\left\{B_{n, \theta}, n \in \mathbb{N}\right\}$ and $\left\{C_{n, \delta} ; n \geq 2\right\}$, respectively.

(a) For any $p \in(0,2)$, we have, as $\epsilon \searrow 0$,

$$
\lambda_{1}(\epsilon, p)=\frac{2}{2-p} \epsilon^{p-2} \sigma^{2}+B_{-\frac{p}{2}} \mathbb{E}\left[|N|^{p}\right] \sigma^{p}+O\left(\epsilon^{p} \log \frac{1}{\epsilon}\right) .
$$

(b) For any $\delta \in(0,1]$, we have, as $\epsilon \searrow 0$,

$$
\lambda_{2}(\epsilon, \delta)=\frac{1}{\delta} \mathbb{E}\left[|N|^{2 \delta+2}\right] \epsilon^{-2 \delta} \sigma^{2 \delta+2}+C_{\delta} \mathbb{E}\left[N^{2}\right] \sigma^{2}+O\left(\epsilon^{2}\right) .
$$

Proposition 2.2 Let $\left\{X, X_{n} ; n \in \mathbb{N}\right\}$ be a sequence of i.i.d. random variables with $\mathbb{E}[X]=0, \mathbb{E}\left[X^{2}\right]=\sigma^{2}>0, \mathbb{E}\left[|X|^{q}\right]<\infty$ for some $q \in(2,3]$, and partial sums $S_{n}=$ $\sum_{k=1}^{n} X_{k}, n \in \mathbb{N}$.

(a) For any $p \in(0,2)$, we have,

$$
\lim _{\epsilon \searrow 0} \epsilon^{\gamma(2-p)} \sum_{n=1}^{\infty} \frac{1}{n^{p}} \int_{\epsilon n}^{\infty} p x^{p-1}\left|\mathbb{P}\left(\left|S_{n}\right| \geq x\right)-\Phi\left(\frac{x}{\sqrt{n}}\right)\right| d x=0,
$$

where $\gamma=\frac{q-p}{2 q-2-p}$.

(b) For any $\delta \in(0,1]$, we have,

$$
\lim _{\epsilon \searrow 0}\left(\log \frac{1}{\epsilon}\right)^{-\delta} \sum_{n=2}^{\infty} \frac{(\log n)^{\delta-1}}{n^{2}} \int_{\epsilon \sqrt{n \log n}}^{\infty} 2 x\left|\mathbb{P}\left(\left|S_{n}\right| \geq x\right)-\Phi\left(\frac{x}{\sqrt{n}}\right)\right| d x=0
$$

Remark 2.5 By Proposotions 4.2, 4.3, 5.2 and 5.3 in Liu and Lin [21], it's easy to get the following results

$$
\begin{gathered}
\lim _{\epsilon \searrow 0} \epsilon^{2-p} \sum_{n=1}^{\infty} \frac{1}{n^{p}} \int_{\epsilon n}^{\infty} p x^{p-1}\left|\mathbb{P}\left(\left|S_{n}\right| \geq x\right)-\Phi\left(\frac{x}{\sqrt{n}}\right)\right| d x=0, \\
\lim _{\epsilon \searrow 0} \epsilon^{2 \delta} \sum_{n=2}^{\infty} \frac{(\log n)^{\delta-1}}{n^{2}} \int_{\epsilon \sqrt{n \log n}}^{\infty} 2 x\left|\mathbb{P}\left(\left|S_{n}\right| \geq x\right)-\Phi\left(\frac{x}{\sqrt{n}}\right)\right| d x=0 .
\end{gathered}
$$

We improve the above results by (2.9) and (2.10) with $0<\gamma<1$. 


\section{Proofs}

In this section, we give the detailed proofs of Theorem 2.1 and Propositions 2.1 and 2.2 . Without loss of generality, we assume $\sigma=1$ in this section. In order to reach our aim, we first introduce some technical lemmas. The first one comes from Billingsley [2].

Lemma 3.1 For $x$ large enough, We have

$$
\Phi(x) \sim \frac{2}{\sqrt{2 \pi} x} e^{-\frac{x^{2}}{2}} .
$$

The following lemma comes from Gut and Steinebach [13] which shows the convergence of the sequence $\left\{B_{n, \theta}, n \in \mathbb{N}\right\}$ for any $\theta \in(-1,0)$.

Lemma 3.2 We have, as $n \rightarrow \infty$,

$$
B_{n, \theta}=B_{\theta}+O\left(n^{\theta}\right), \quad \text { for }-1<\theta<0,
$$

where $B_{n, \theta}$ is defined by (2.1) and $B_{\theta}$ is a constant with $-\frac{1}{\theta+1} \leq B_{\theta}<\frac{\theta}{\theta+1}<0$.

Similar to Lemma 3.2, the following lemma shows the convergence of the sequence $\left\{C_{n, \delta}, n \geq 2\right\}$ for any $\delta \in(0,1]$.

Lemma 3.3 For any $0<\delta \leq 1$, we have, as $n \rightarrow \infty$,

$$
C_{n, \delta}=C_{\delta}+O\left(\frac{(\log n)^{\delta-1}}{n}\right)
$$

where $C_{n, \delta}$ is defined by (2.2) and $C_{\delta}$ is a constant with $-\frac{(\log 2)^{\delta}}{\delta} \leq C_{\delta} \leq 0$.

Proof: It follows from (2.2) and the mean value theorem that

$$
\begin{aligned}
C_{n+1, \delta}-C_{n, \delta}= & \frac{[\log (n+1)]^{\delta-1}}{n+1}-\frac{[\log (n+1)]^{\delta}-(\log n)^{\delta}}{\delta} \\
& =\frac{[\log (n+1)]^{\delta-1}}{n+1}-\frac{\left(\log \xi_{n}\right)^{\delta-1}}{\xi_{n}}
\end{aligned}
$$

for some $\xi_{n} \in(n, n+1)$.

On the other hand, noting

$$
C_{n, \delta}=\sum_{j=2}^{n} \int_{j-1}^{j}\left[\frac{(\log j)^{\delta-1}}{j}-\frac{(\log x)^{\delta-1}}{x}\right] d x,
$$

we get that

$$
\begin{aligned}
0 \geq C_{n, \delta} & \geq \sum_{j=3}^{n}\left[\frac{(\log j)^{\delta-1}}{j}-\frac{(\log (j-1))^{\delta-1}}{j-1}\right]+\frac{(\log 2)^{\delta-1}}{2}-\int_{1}^{2} \frac{(\log x)^{\delta-1}}{x} d x \\
& =\frac{(\log n)^{\delta-1}}{n}-\frac{(\log 2)^{\delta}}{\delta} \geq-\frac{(\log 2)^{\delta}}{\delta}
\end{aligned}
$$

since $f(x)=\frac{(\log x)^{\delta-1}}{x}$ is a decreasing function. 
Since the function $f(x)=\frac{(\log x)^{\delta-1}}{x}$ is decreasing with $\delta \in(0,1]$, we obtain that the sequence $\left\{C_{n, \delta}, n \geq 2\right\}$ is also decreasing. By using this fact, we get from the monotone bounded theorem that $\lim _{n \rightarrow \infty} C_{n, \delta}$ exists, since (3.4) implies that $\left\{C_{n, \delta}, n \geq 2\right\}$ is a bounded sequence.

For any $\delta \in(0,1]$, let $C_{\delta}$ denote the limit of the sequence $\left\{C_{n, \delta}, n \geq 2\right\}$. Moreover, from (3.4) we get that $-\frac{(\log 2)^{\delta}}{\delta} \leq C_{\delta} \leq 0$.

Given $m>n$, using the mean value theorem again, we have

$$
\begin{aligned}
0>C_{m, \delta}-C_{n, \delta} & =\sum_{j=n+1}^{m}\left[\frac{(\log j)^{\delta-1}}{j}-\frac{(\log j)^{\delta}-(\log (j-1))^{\delta}}{\delta}\right] \\
& =\sum_{j=n+1}^{m}\left[\frac{(\log j)^{\delta-1}}{j}-\frac{\left(\log \xi_{j}\right)^{\delta-1}}{\xi_{j}}\right] \quad \text { for some } \xi_{j} \in(j-1, j) \\
& >\sum_{j=n+1}^{m}\left[\frac{(\log j)^{\delta-1}}{j}-\frac{(\log (j-1))^{\delta-1}}{j-1}\right] \\
& =\frac{(\log m)^{\delta-1}}{m}-\frac{(\log n)^{\delta-1}}{n} .
\end{aligned}
$$

Letting $m \rightarrow \infty$ in (3.5), we finish the proof of Lemma 3.3 .

Now we come to a point where we can prove the Propositions 2.1 and 2.2. Next, we first prove the Proposition 2.1.

Proof of Proposition [2.1: Let $\left\{X, X_{n}, n \in \mathbb{N}\right\}$ be a sequence of i.i.d. normal random variables with mean 0 and variance $\sigma^{2}>0$. Since we assume $\sigma=1$, we indeed deal with the standard normal random variable $N$.

We first prove the part (a) of Proposition 2.1. Note that, for $0<p<2$,

$$
\lambda_{1}(\epsilon, p)=\epsilon^{p} \sum_{n=1}^{\infty} \mathbb{P}\left(\left|S_{n}\right| \geq \epsilon n\right)+\sum_{n=1}^{\infty} \frac{1}{n^{p}} \int_{\epsilon n}^{\infty} p x^{p-1} \mathbb{P}\left(\left|S_{n}\right| \geq x\right) d x .
$$

On the other hand, from Klesov [17, we have

$$
\sum_{n=1}^{\infty} \mathbb{P}\left(\left|S_{n}\right| \geq \epsilon n\right)=\frac{\sigma^{2}}{\epsilon^{2}}-\frac{1}{2}+o(1) .
$$

Thus, in order to prove (3.6), we only need to consider the second term in (3.6).

The change of variable $t=\frac{x}{\sqrt{n}}$ yields that

$$
\begin{aligned}
\frac{1}{n^{p}} \int_{\epsilon n}^{\infty} p x^{p-1} \mathbb{P}\left(\left|S_{n}\right| \geq x\right) d x & =\frac{1}{n^{\frac{p}{2}}} \int_{\epsilon \sqrt{n}}^{\infty} p t^{p-1} \Phi(t) d t \\
& =\frac{1}{n^{\frac{p}{2}}} \sum_{j=n}^{\infty} \int_{\epsilon \sqrt{j}}^{\epsilon \sqrt{j+1}} p t^{p-1} \Phi(t) d t
\end{aligned}
$$

since

$$
\frac{S_{n}}{\sqrt{n}} \stackrel{d}{=} N
$$


and

$$
\Phi(t)=\mathbb{P}(|N| \geq t)
$$

It follows from the Fubini's theorem, (3.8) and Lemma 3.2 that

$$
\begin{aligned}
& \sum_{n=1}^{\infty} \frac{1}{n^{p}} \int_{\epsilon n}^{\infty} p x^{p-1} \mathbb{P}\left(\left|S_{n}\right| \geq x\right) d x \\
= & \sum_{j=1}^{\infty}\left(\sum_{n=1}^{j} \frac{1}{n^{\frac{p}{2}}}\right) \int_{\epsilon \sqrt{j}}^{\epsilon \sqrt{j+1}} p t^{p-1} \Phi(t) d t \\
= & I_{31}+I_{32}+I_{33},
\end{aligned}
$$

where

$$
\begin{aligned}
& I_{31}=\frac{2 p}{2-p} \sum_{j=1}^{\infty} j^{-\frac{p}{2}+1} \int_{\epsilon \sqrt{j}}^{\epsilon \sqrt{j+1}} t^{p-1} \Phi(t) d t \\
& I_{32}=B_{-\frac{p}{2}} \sum_{j=1}^{\infty} \int_{\epsilon \sqrt{j}}^{\epsilon \sqrt{j+1}} p t^{p-1} \Phi(t) d t \\
& I_{33}=\sum_{j=1}^{\infty} O\left(j^{-\frac{p}{2}}\right) \sum_{j=1}^{\infty} \int_{\epsilon \sqrt{j}}^{\epsilon \sqrt{j+1}} p t^{p-1} \Phi(t) d t .
\end{aligned}
$$

Now, we deal with $I_{31}$. The integer mean theorem implies that for some $\xi \in(j, j+1)$

$$
\int_{\epsilon \sqrt{j}}^{\epsilon \sqrt{j+1}} t^{p-1} \Phi(t) d t=\epsilon^{p} \xi^{\frac{p-1}{2}} \Phi(\epsilon \sqrt{\xi})(\sqrt{j+1}-\sqrt{j}) .
$$

Furthermore, by the Taylor expansion, we have

$$
\begin{aligned}
\xi^{\frac{p-1}{2}} & =j^{\frac{p-1}{2}}+O\left(j^{\frac{p-3}{2}}\right), \\
\Phi(\epsilon \sqrt{\xi}) & =\Phi(\epsilon \sqrt{j})+\epsilon e^{-\frac{\epsilon^{2} j}{2}} O\left(j^{-\frac{1}{2}}\right), \\
\sqrt{j+1}-\sqrt{j} & =\frac{1}{2} j^{-\frac{1}{2}}+O\left(j^{-\frac{3}{2}}\right) .
\end{aligned}
$$

From the above argument, we have

$$
I_{31}=\frac{p \epsilon^{p}}{2-p} \sum_{j=1}^{\infty}\left[\Phi(\epsilon \sqrt{j})+\epsilon e^{-\frac{\epsilon^{2} j}{2}} O\left(j^{-\frac{1}{2}}\right)+\Phi(\epsilon \sqrt{j}) O\left(j^{-1}\right)+O\left(j^{-\frac{3}{2}}\right)\right] .
$$

Letting $y=\epsilon \sqrt{t}$, we have

$$
\begin{aligned}
\frac{p \epsilon^{p}}{2-p} \sum_{j=1}^{\infty} \Phi(\epsilon \sqrt{j})= & \frac{p \epsilon^{p}}{2-p} \int_{1}^{\infty} \Phi(\epsilon \sqrt{t}) d t+O\left(\epsilon^{p}\right) \\
& =\frac{p \epsilon^{p-2}}{2-p} \int_{\epsilon}^{\infty} \Phi(y) 2 y d y+O\left(\epsilon^{p}\right) \\
& =\frac{p \epsilon^{p-2} \mathbb{E}\left[N^{2}\right]}{2-p}-\frac{p \epsilon^{p-2}}{2-p} \int_{0}^{\epsilon} 2 t \Phi(y) d y+O\left(\epsilon^{p}\right) \\
& =\frac{p \epsilon^{p-2} \mathbb{E}\left[N^{2}\right]}{2-p}+O\left(\epsilon^{p}\right) .
\end{aligned}
$$


Similar to (3.11), we can obtain that

$$
\begin{gathered}
\frac{p \epsilon^{p+1}}{2-p} \sum_{j=1}^{\infty} O\left(j^{-\frac{1}{2}}\right) e^{-\frac{\epsilon^{2} j}{2}}=O\left(\epsilon^{p}\right), \\
\frac{p \epsilon^{p}}{2-p} \sum_{j=1}^{\infty} O\left(j^{-1}\right) \Phi(\epsilon \sqrt{j})=O\left(\epsilon^{p} \log \frac{1}{\epsilon}\right),
\end{gathered}
$$

and

$$
\frac{p \epsilon^{p}}{2-p} \sum_{j=1}^{\infty} O\left(j^{-\frac{3}{2}}\right)=O\left(\epsilon^{p}\right)
$$

From (3.11) to (3.14), we have

$$
I_{31}=\frac{p \epsilon^{p-2} \mathbb{E}\left[N^{2}\right]}{2-p}+O\left(\epsilon^{p} \log \frac{1}{\epsilon}\right) .
$$

For $I_{32}$, we have

$$
\begin{aligned}
I_{32} & =B_{-\frac{p}{2}} \int_{\epsilon}^{\infty} p t^{p-1} \Phi(t) d t \\
& =B_{-\frac{p}{2}} \mathbb{E}\left[|N|^{p}\right]-B_{-\frac{p}{2}} \int_{0}^{\epsilon} p t^{p-1} \Phi(t) d t \\
& =B_{-\frac{p}{2}} \mathbb{E}\left[|N|^{p}\right]+O\left(\epsilon^{p}\right) .
\end{aligned}
$$

Next, we deal with $I_{33}$. Noting that

$$
(j+1)^{\frac{p}{2}}-j^{\frac{p}{2}}=O\left(j^{\frac{p}{2}-1}\right), \text { as } j \rightarrow \infty,
$$

we have

$$
\int_{\epsilon \sqrt{j}}^{\epsilon \sqrt{j+1}} p t^{p-1} \Phi(t) d t \leq \epsilon^{p} \Phi(\epsilon \sqrt{j})\left[(j+1)^{\frac{p}{2}}-j^{\frac{p}{2}}\right]=\epsilon^{p} \Phi(\epsilon \sqrt{j}) O\left(j^{\frac{p}{2}-1}\right),
$$

since $\Phi(t)$ is a decreasing function.

It follows from (3.17) that

$$
\begin{aligned}
I_{33} & \leq C \epsilon^{p} \sum_{j=1}^{\infty} j^{-1} \Phi(\epsilon \sqrt{j}) \\
& =C \epsilon^{p} \int_{1}^{\infty} \frac{\Phi(\epsilon \sqrt{x})}{x} d x+O\left(\epsilon^{p}\right)=C \epsilon^{p} \int_{\epsilon}^{\infty} \frac{\Phi(t)}{t} d t+O\left(\epsilon^{p}\right) \\
& \leq C \epsilon^{p} \int_{\epsilon}^{1} t^{-1} d t+C \epsilon^{p} \int_{1}^{\infty} \Phi(t) d t+O\left(\epsilon^{p}\right)=O\left(\epsilon^{p} \log \frac{1}{\epsilon}\right),
\end{aligned}
$$

where the second equation in (3.18) follows from the change of variable $t=\epsilon \sqrt{x}$ again. (3.15), (3.16) and (3.19) imply that

$$
\sum_{n=1}^{\infty} \frac{1}{n^{p}} \int_{\epsilon n}^{\infty} p x^{p-1} \mathbb{P}\left(\left|S_{n}\right| \geq x\right) d x=\frac{p \epsilon^{p-2} \mathbb{E}\left[N^{2}\right]}{2-p}+B_{-\frac{p}{2}} \mathbb{E}\left[|N|^{p}\right]+O\left(\epsilon^{p} \log \frac{1}{\epsilon}\right) .
$$


From (3.6), (3.7) and (3.20), we get the part (a) in Proposition 2.1.

Below, we prove the part (b). The proof of this part is similar to that of the part (a). However, some modifications are needed to characterize the lower bound $\epsilon \sqrt{n \log n}$. Note that, for any $\delta \in(0,1]$,

$$
\begin{aligned}
\lambda_{2}(\epsilon, \delta)= & \epsilon^{2} \sum_{n=2}^{\infty} \frac{(\log n)^{\delta}}{n} \mathbb{P}\left(\left|S_{n}\right| \geq \epsilon \sqrt{n \log n}\right)+ \\
& \sum_{n=2}^{\infty} \frac{(\log n)^{\delta-1}}{n^{2}} \int_{\epsilon \sqrt{n \log n}}^{\infty} 2 x \mathbb{P}\left(\left|S_{n}\right| \geq x\right) d x .
\end{aligned}
$$

It follows from Kong [18] that

$$
\sum_{n=2}^{\infty} \frac{(\log n)^{\delta}}{n} \mathbb{P}\left(\left|S_{n}\right| \geq \epsilon \sqrt{n \log n}\right)=\frac{\epsilon^{-2 \delta-2} \mathbb{E}\left[|N|^{2 \delta+2}\right]}{\delta+1}+O(1)
$$

Hence, we only need to compute the second term in (3.21). In fact, by the Fubini's theorem and Lemma 3.3, we have

$$
\begin{aligned}
& \sum_{n=2}^{\infty} \frac{(\log n)^{\delta-1}}{n^{2}} \int_{\epsilon \sqrt{n \log n}}^{\infty} 2 x \mathbb{P}\left(\left|S_{n}\right| \geq x\right) d x \\
= & \sum_{j=2}^{\infty}\left(\sum_{n=2}^{j} \frac{(\log n)^{\delta-1}}{n}\right) \int_{\epsilon \sqrt{\log j}}^{\epsilon \sqrt{\log (j+1)}} 2 t \Phi(t) d t \\
= & : I_{34}+I_{35}+I_{36},
\end{aligned}
$$

where

$$
\begin{aligned}
& I_{34}=\frac{1}{\delta} \sum_{j=2}^{\infty}(\log j)^{\delta} \int_{\epsilon \sqrt{\log j}}^{\epsilon \sqrt{\log (j+1)}} 2 t \Phi(t) d t, \\
& I_{35}=C_{\delta} \sum_{j=2}^{\infty} \int_{\epsilon \sqrt{\log j}}^{\epsilon \sqrt{\log (j+1)}} 2 t \Phi(t) d t, \\
& \left.I_{36}=\sum_{j=2}^{\infty} O\left(\frac{(\log j)^{\delta-1}}{j}\right) \int_{\epsilon \sqrt{\log j}}^{\epsilon \sqrt{\log (j+1)}} 2 t \Phi(t)\right) d t .
\end{aligned}
$$

We first consider $I_{34}$. The integer mean theorem shows that

$$
\int_{\epsilon \sqrt{\log j}}^{\epsilon \sqrt{\log (j+1)}} 2 t \Phi(t) d t=\epsilon^{2} \Phi(\epsilon \sqrt{\log \xi})(\log (j+1)-\log j) \text { for some } \xi \in(j, j+1)
$$

On the other hand, it follows from the Taylor expansion that

$$
\begin{aligned}
\Phi(\epsilon \sqrt{\log \xi}) & =\Phi(\epsilon \sqrt{\log j})+\epsilon O\left(j^{-1-\frac{\epsilon^{2}}{2}}(\log j)^{-\frac{1}{2}}\right), \\
\log (j+1)-\log j & =j^{-1}+O\left(j^{-2}\right) .
\end{aligned}
$$


By the above argument, we have

$$
I_{34}=\frac{\epsilon^{2}}{\delta} \sum_{j=2}^{\infty} \frac{(\log j)^{\delta}}{j} \Phi(\epsilon \sqrt{\log j})+O\left(\epsilon^{2}\right) .
$$

Putting $t=\epsilon \sqrt{\log x}$, we have

$$
\begin{aligned}
& \frac{\epsilon^{2}}{\delta} \int_{2}^{\infty} \frac{(\log x)^{\delta}}{x} \Phi(\epsilon \sqrt{\log x}) d x \\
= & \frac{2 \epsilon^{-2 \delta}}{\delta} \int_{\epsilon \sqrt{\log 2}}^{\infty} t^{2 \delta+1} \Phi(t) d t=\frac{\epsilon^{-2 \delta} \mathbb{E}\left[|N|^{2 \delta+2}\right]}{\delta(\delta+1)}+O\left(\epsilon^{2}\right),
\end{aligned}
$$

since

$$
\int_{0}^{\epsilon \sqrt{\log 2}} 2 t^{2 \delta+1} \Phi(t) d t \leq \int_{0}^{\epsilon \sqrt{\log 2}} 2 t^{2 \delta+1} d t=O\left(\epsilon^{2 \delta+2}\right) .
$$

It follows from (3.25) that

$$
I_{34} \leq \frac{\epsilon^{-2 \delta} \mathbb{E}\left[|N|^{2 \delta+2}\right]}{\delta(\delta+1)}+O\left(\epsilon^{2}\right)
$$

For $I_{35}$, we have

$$
I_{35}=C_{\delta} \int_{0}^{\infty} 2 t \Phi(t) d t-C_{\delta} \int_{0}^{\epsilon \sqrt{\log 2}} 2 t \Phi(t) d t=C_{\delta} \mathbb{E}\left[N^{2}\right]+O\left(\epsilon^{2}\right) .
$$

Finally, we look at $I_{36}$. By (3.24), we have

$$
I_{36} \leq C \epsilon^{2} \sum_{j=2}^{\infty} \frac{(\log j)^{\delta-1}}{j^{2}} \Phi(\epsilon \sqrt{\log j})+O\left(\epsilon^{2}\right)=O\left(\epsilon^{2}\right)
$$

where

$$
0<\delta \leq 1, \text { and } \Phi(\epsilon \sqrt{\log j}) \leq 1 \text { for any } j \geq 2 .
$$

Hence, from (3.26) to (3.28), we obtain

$$
\begin{aligned}
& \sum_{n=2}^{\infty} \frac{(\log n)^{\delta-1}}{n^{2}} \int_{\epsilon \sqrt{n \log n}}^{\infty} 2 x \mathbb{P}\left(\left|S_{n}\right| \geq x\right) d x \\
& \quad=\frac{\epsilon^{-2 \delta} \mathbb{E}\left[|N|^{2 \delta+2}\right]}{\delta(\delta+1)}+C_{\delta} \mathbb{E}\left[N^{2}\right]+O\left(\epsilon^{2}\right) .
\end{aligned}
$$

Therefore, we get from (3.21), (3.22) and (3.29) that the part (b) holds. The proof of this proposition is finished.

Next, we focus on the proof of Proposition 2.2.

Proof of Proposition 2.2: Let $\left\{X, X_{n} ; n \geq 1\right\}$ be a sequence of i.i.d. random variables with mean zero, $\mathbb{E}\left[X^{2}\right]=1$ and $\mathbb{E}\left[|X|^{q}\right]<\infty$ for some $q \in(2,3]$. Moreover, for any $n \in \mathbb{N}$, set

$$
\Delta_{n}:=\sup _{t}\left|\mathbb{P}\left(\left|S_{n}\right| \geq \sqrt{n} t\right)-\Phi(t)\right|
$$


We first prove the part (a). For any $M \geq 1$, let

$$
H_{1}(\epsilon)=M \epsilon^{-2 \gamma},
$$

where $\gamma=\frac{q-p}{2 q-2-p}>0$ with $0<p<2$ and $2<q \leq 3$.

By the change of variable $t=\frac{x}{\sqrt{n}}$, we have

$$
\begin{aligned}
\sum_{n=1}^{\infty} n^{-p} \int_{\epsilon n}^{\infty} p x^{p-1}\left|\mathbb{P}\left(\left|S_{n}\right| \geq x\right)-\Phi\left(\frac{x}{\sqrt{n}}\right)\right| d x \\
\quad=\sum_{n=1}^{\infty} n^{-\frac{p}{2}} \int_{\epsilon \sqrt{n}}^{\infty} p t^{p-1}\left|\mathbb{P}\left(\left|S_{n}\right| \geq \sqrt{n} t\right)-\Phi(t)\right| d t
\end{aligned}
$$

Based on $H_{1}(\epsilon)$, we split the right hand side of (3.31) into two parts. We first observe the first part, that is,

$$
\sum_{n \leq H_{1}(\epsilon)} n^{-\frac{p}{2}} \int_{\epsilon \sqrt{n}}^{\infty} p t^{p-1}\left|\mathbb{P}\left(\left|S_{n}\right| \geq \sqrt{n} t\right)-\Phi(t)\right| d t \leq \sum_{n \leq H_{1}(\epsilon)} n^{-\frac{p}{2}}\left(J_{1}+J_{2}\right),
$$

where

$$
J_{1}=\int_{0}^{\Delta_{n}^{-\frac{1}{2 p}}} p t^{p-1}\left|\mathbb{P}\left(\left|S_{n}\right| \geq \sqrt{n} t\right)-\Phi(t)\right| d t
$$

and

$$
J_{2}=\int_{\Delta_{n}^{-\frac{1}{2 p}}}^{\infty} p t^{p-1}\left|\mathbb{P}\left(\left|S_{n}\right| \geq \sqrt{n} t\right)-\Phi(t)\right| d t
$$

It follows from

$$
\frac{S_{n}}{\sqrt{n}} \stackrel{d}{\rightarrow} N
$$

that

$$
\Delta_{n} \rightarrow 0
$$

as $n \rightarrow \infty$. Thus,

$$
J_{1} \leq \Delta_{n} \int_{0}^{\Delta_{n}^{-\frac{1}{2 p}}} p t^{p-1} d t=\Delta_{n}^{\frac{1}{2}} \rightarrow 0 \quad(n \rightarrow \infty)
$$

On the other hand, it follows from $\frac{S_{n}}{\sqrt{n}} \stackrel{d}{\rightarrow} N$ and Lemma 3.1 that for large enough $t$,

$$
\left|\mathbb{P}\left(\left|S_{n}\right| \geq \sqrt{n} t\right)-\Phi(t)\right| \leq C e^{-\frac{t^{2}}{2}} t^{-1} \leq C e^{-t} t^{1-p} .
$$

Hence, as $n$ goes to $\infty$,

$$
J_{2} \leq C \int_{\Delta_{n}^{-\frac{1}{2 p}}}^{\infty} e^{-t} d t \rightarrow 0 .
$$

By the Toeplitz lemma, (3.33) and (3.35), we have that for any $M \geq 1$,

$$
\lim _{\epsilon \searrow 0} \epsilon^{\gamma(2-p)} \sum_{n \leq H_{1}(\epsilon)} n^{-\frac{p}{2}}\left(J_{1}+J_{2}\right)=\lim _{\epsilon \searrow 0} \frac{M^{1-\frac{p}{2}}}{\left(H_{1}(\epsilon)\right)^{1-\frac{p}{2}}} \sum_{n \leq H_{1}(\epsilon)} n^{-\frac{p}{2}}\left(J_{1}+J_{2}\right)=0 .
$$


Next, we look at the second part. Recall that Bikjalis [3] got the following non-uniform large deviation estimate, for any $x>0$,

$$
\left|\mathbb{P}\left(S_{n}>\sqrt{n} x\right)-\mathbb{P}(N>x)\right| \leq \frac{C \mathbb{E}\left[|X|^{q}\right]}{n^{\frac{q}{2}-1}\left(1+x^{q}\right)}, \quad 2<q \leq 3 .
$$

Hence

$$
\begin{aligned}
\sum_{n \geq H_{1}(\epsilon)} n^{-\frac{p}{2}} \int_{\epsilon \sqrt{n}}^{\infty} p t^{p-1}\left|\mathbb{P}\left(\left|S_{n}\right| \geq \sqrt{n} t\right)-\Phi(t)\right| d t \\
\quad \leq \sum_{n \geq H_{1}(\epsilon)} n^{-\frac{p}{2}} \int_{\epsilon \sqrt{n}}^{\infty} \frac{C t^{p-1}}{n^{\frac{q}{2}-1}\left(1+t^{q}\right)} d t \\
\quad \leq \sum_{n \geq H_{1}(\epsilon)} C n^{1-\frac{p}{2}-\frac{q}{2}} \int_{\epsilon \sqrt{n}}^{\infty} t^{p-q-1} d t=C \epsilon^{p-q} \sum_{n \geq H_{1}(\epsilon)} n^{1-q}
\end{aligned}
$$

Thus,

$$
\begin{aligned}
\limsup _{\epsilon \searrow 0} \epsilon^{\gamma(2-p)} \sum_{n \geq H_{1}(\epsilon)} n^{-\frac{p}{2}} \int_{\epsilon \sqrt{n}}^{\infty} p t^{p-1}\left|\mathbb{P}\left(\left|S_{n}\right| \geq \sqrt{n} t\right)-\Phi(t)\right| d t \\
\leq C \underset{\epsilon \searrow 0}{\lim \sup } \epsilon^{\gamma(2-p)+(p-q)}\left(H_{1}(\epsilon)\right)^{2-q} \\
=C M^{2-q} \searrow 0, \text { as } M \nearrow \infty
\end{aligned}
$$

since $q>2$.

Finally, we get from (3.36) and (3.38) that the part (a).

Below, we prove the part (b). In fact, the proof of this part is similar to that of part (a) and we only write the modifications in the following.

For any $M \geq 1$, define

$$
H_{2}(\epsilon)=M \epsilon^{-2}\left(\log \frac{1}{\epsilon}\right)^{\frac{2 \delta}{2-q}}
$$

It is obvious that as $\epsilon \searrow 0$,

$$
H_{2}(\epsilon) \nearrow \infty .
$$

The change of variable $t=\frac{x}{\sqrt{n}}$ yields that

$$
\begin{aligned}
\sum_{n=2}^{\infty} \frac{(\log n)^{\delta-1}}{n^{2}} \int_{\epsilon \sqrt{n \log n}}^{\infty} 2 x\left|\mathbb{P}\left(\left|S_{n}\right| \geq x\right)-\Phi\left(\frac{x}{\sqrt{n}}\right)\right| d x \\
=\sum_{n=2}^{\infty} \frac{(\log n)^{\delta-1}}{n} \int_{\epsilon \sqrt{\log n}}^{\infty} 2 t\left|\mathbb{P}\left(\left|S_{n}\right| \geq \sqrt{n} t\right)-\Phi(t)\right| d t
\end{aligned}
$$

According to $H_{2}(\epsilon)$, we split the sum in (3.39) into two part. For the first part,

$$
\sum_{n=2}^{H_{2}(\epsilon)} \frac{(\log n)^{\delta-1}}{n} \int_{\epsilon \sqrt{\log n}}^{\infty} 2 t\left|\mathbb{P}\left(\left|S_{n}\right| \geq \sqrt{n} t\right)-\Phi(t)\right| d t \leq \sum_{n=2}^{H_{2}(\epsilon)} \frac{(\log n)^{\delta-1}}{n}\left(J_{3}+J_{4}\right),
$$


where

$$
J_{3}=\int_{0}^{\Delta_{n}^{-\frac{1}{4}}} 2 t\left|\mathbb{P}\left(\left|S_{n}\right| \geq \sqrt{n} t\right)-\Phi(t)\right| d t
$$

and

$$
J_{4}=\int_{\Delta_{n}^{-\frac{1}{4}}}^{\infty} 2 t\left|\mathbb{P}\left(\left|S_{n}\right| \geq \sqrt{n} t\right)-\Phi(t)\right| d t
$$

with $\Delta_{n}$ being defined by (3.30). Similar to (3.33) and (3.35), we obtain that $J_{3}$ and $J_{4}$ goes to 0 as $n \rightarrow \infty$, respectively. Thus, by using the Toeplitz lemma, we have, for any $M \geq 1$

$$
\begin{aligned}
& \lim _{\epsilon \searrow 0}\left(\log \frac{1}{\epsilon}\right)^{-\delta} \sum_{n=2}^{H_{2}(\epsilon)} \frac{(\log n)^{\delta-1}}{n}\left(J_{3}+J_{4}\right) \\
& \quad=\lim _{\epsilon \searrow 0} \frac{2^{\delta}}{\left[\log H_{2}(\epsilon)\right]^{\delta}} \sum_{n=2}^{H_{2}(\epsilon)} \frac{(\log n)^{\delta-1}}{n}\left(J_{3}+J_{4}\right)=0 .
\end{aligned}
$$

Furthermore, by using the large deviation (3.37), we have

$$
\begin{aligned}
\sum_{n \geq H_{2}(\epsilon)} \frac{(\log n)^{\delta-1}}{n} \int_{\epsilon \sqrt{\log n}}^{\infty} 2 t\left|\mathbb{P}\left(\left|S_{n}\right| \geq \sqrt{n} t\right)-\Phi(t)\right| d t \\
\leq \sum_{n \geq H_{2}(\epsilon)} \frac{(\log n)^{\delta-1}}{n^{\frac{q}{2}}} \int_{\epsilon \sqrt{\log n}}^{\infty} \frac{C t \mathbb{E}\left[|X|^{q}\right]}{1+t^{q}} d t \\
=C \epsilon^{2-q} \sum_{n \geq H_{2}(\epsilon)}(\log n)^{\delta-\frac{q}{2}} n^{-\frac{q}{2}} .
\end{aligned}
$$

Note that

$$
0<\delta \leq 1 \text { and } 2<q \leq 3 .
$$

Hence, we have $\delta-\frac{q}{2}<0$ and

$$
\begin{aligned}
\limsup _{\epsilon \searrow 0}\left(\log \frac{1}{\epsilon}\right)^{-\delta} \sum_{n \geq H_{2}(\epsilon)} \frac{(\log n)^{\delta-1}}{n} \int_{\epsilon \sqrt{\log n}}^{\infty} 2 t\left|\mathbb{P}\left(\left|S_{n}\right| \geq \sqrt{n} t\right)-\Phi(t)\right| d t \\
\quad \leq C \limsup _{\epsilon \searrow 0}\left(\log \frac{1}{\epsilon}\right)^{-\delta} \epsilon^{2-q} \sum_{n \geq H_{2}(\epsilon)} n^{-\frac{q}{2}} \\
\leq C \limsup _{\epsilon \searrow 0}\left(\log \frac{1}{\epsilon}\right)^{-\delta} \epsilon^{2-q}\left(H_{2}(\epsilon)\right)^{1-\frac{q}{2}} \\
\leq C M^{1-\frac{q}{2}} \searrow 0, \text { as } M \nearrow \infty .
\end{aligned}
$$

Thus, we get from (3.40) and (3.40) the part (b) of Proposition 2.2 .

Finally, we prove the main result of this paper.

Proof of Theorem 2.1: In order to prove it, we first should point out that (3.6) and (3.21) also hold under the conditions of Theorem 2.1. We prove this theorem by two steps. 
We first prove the part (a) of this theorem. Recall that He and Xie [14] obtained that

$$
\sum_{n=1}^{\infty} \mathbb{P}\left(\left|S_{n}\right| \geq \epsilon n\right)=\epsilon^{-2}\left(\sigma^{2}+o\left(\epsilon^{q-2}\right)\right) .
$$

Combing (3.6) and (3.42), we get that for any $0<p<2$

$$
\lambda_{1}(\epsilon, p)=\sigma^{2} \epsilon^{p-2}+\epsilon^{p+q-4} o(1)+\sum_{n=1}^{\infty} n^{-p} \int_{\epsilon n}^{\infty} p x^{p-1} \mathbb{P}\left(\left|S_{n}\right| \geq x\right) d x .
$$

Thus, by (3.20), we have

$$
\begin{aligned}
\lambda_{1}(\epsilon, p)=\frac{2 \sigma^{2} \epsilon^{p-2}}{2-p} & +B_{-\frac{p}{2}} \sigma^{p} \mathbb{E}\left[|N|^{p}\right]+\epsilon^{p+q-4} o(1)+O\left(\epsilon^{p} \log \frac{1}{\epsilon}\right) \\
& +\sum_{n=1}^{\infty} n^{-p} \int_{\epsilon n}^{\infty} p x^{p-1}\left[\mathbb{P}\left(\left|S_{n}\right| \geq x\right)-\Phi\left(\frac{x}{\sqrt{n}}\right)\right] d x
\end{aligned}
$$

Hence, combing (3.43) and part (a) of Proposition 2.2 together, we get that

$$
\lim _{\epsilon \searrow 0} \epsilon^{\gamma(2-p)}\left[\lambda_{1}(\epsilon, p)-\frac{2 \sigma^{2} \epsilon^{p-2}}{2-p}\right]=0
$$

where

$$
0<p<2, \quad 0<\gamma=\frac{q-p}{2 q-2-p}<1
$$

and

$$
\gamma(2-p)+p+q-4=\frac{2(q-2)^{2}}{2 q-2-p}>0 .
$$

Hence the part (a) holds.

Next, we prove the part (b). The arguments for the part (b) are similar to those for the part (a). Kong [18] got that (3.22) also holds for all random variables satisfying (2.3). Thus, by (3.29) and part (b) of Proposition 2.2, we get that

$$
\lim _{\epsilon \searrow 0}\left(\log \frac{1}{\epsilon}\right)^{-\delta}\left[\lambda_{2}(\epsilon, \delta)-\frac{\sigma^{2 \delta+2}}{\delta} \mathbb{E}\left[|N|^{2 \delta+2}\right] \epsilon^{-2 \delta}\right]=0 .
$$

Therefore, we get part (b). The proof of Theorem 2.1 is finished.

Acknowledgments: This work was supported by the Natural Science Foundation of China (No.11361007), the Guangxi Natural Science Foundation (Nos.2012GXNSFBA053010 and 2014GXNSFCA118001) and the Project for Fostering Distinguished Youth Scholars of Shandong University of Finance and Economics.

\section{References}

[1] L. E. Baum, M. Katz. (1965). Convergence rates in the law of large numbers. Trans. Amer. Math. Soc. 120, 108-123.

[2] P. Billingsley. (1999). Convergence of Probability Measures, Second Edition. Wiley, New York. 
[3] A. Bikjalis [A. Bikelis]. (1966). Estimates of the remainder term in the central limit theorem. (Russian. Lithuanian and English summaries.) Litovsk. Mat. Sb. 6, 323-346.

[4] R. Chen. (1978). A remark on the tail probability of a distribution. J. Multivariate Analysis 8, 328-333.

[5] J. A. Davis. (1968). Convergence rates for the law of the iterated logarithm. Ann. Math. Statist. 39, 1479-1485.

[6] J. A. Davis. (1968). Convergence rates for probabilities of moderate deviations. Ann. Math. Statist. 39, 2016-2028.

[7] P. Erdős. (1949). On a theorem of Hsu and Robbins. Ann. Math. Statist. 20, 286-291.

[8] P. Erdős. (1950). Remark on my paper "On a theorem of Hsu and Robbins". Ann. Math. Statist. 21, 138.

[9] A. Gut. (1980). Convergence rates for Probabilities of moderate deviations for sums of random variables with multidimensional indices. Ann. Probab. 8, 298-313.

[10] A. Gut, A. Spătaru. (2000). Precise asymptotics in the Baum-Katz and Davis law of large numbers. J. Math. Anal. Appl. 248, 233-246.

[11] A. Gut, A. Spătaru. (2000). Precise asymptotics in the law of the iterated logarithm. Ann. Probab. 28, 1870-1883.

[12] A. Gut, J. Steinebach. (2012). Convergence rates in precise asymptotics. J. Math. Anal. Appl. 390, 1-14.

[13] A. Gut, J. Steinebach. (2013). Convergence rates in precise asymptotics II. Annales Univ. Sci. Budapest., Sect. Comp. 39, 95-110.

[14] J. J. He, T. F. Xie. (2013). Asymptotic property for some series of probability. Acta Math. Appl. Sin. 29, 179-186.

[15] C. C. Heyde. (1975). A supplement to the strong law of large numbers. J. Appl. Probab. 12, 173-175.

[16] P. L. Hsu, H. Robbins. (1947). Complete convergence and the law of large numbers. Proc. Nat. Acad. Sci. U.S.A. 33, 25-31.

[17] O. I. Klesov. (1994). On the convergence rate in a theorem of Heyde. Theory Probab. Math. Statist. 49, 83-87.

[18] L. T. Kong. (2015). Convergence rate in precise asymptotics for Davis law of large numbers. arXiv:1507.06406.

[19] T. L. Lai. (1974). Limit theorems for delayed sums. Ann. Probab. 2, 432-440.

[20] D. L. Li, A. Spătaru. (2012). Asymptotics related to a series of T.L. Lai. Statist. Probab. Letters 82, 1538-1548. 
[21] W. D. Liu, Z. Y. Lin. (2006). Precise asymptotics for a new kind of complete moment convergence. Statist. Probab. Letters 76, 1787-1799.

[22] A. Spătaru. (2004). Precise asymptotics for a series of T.L. Lai. Proc. Amer. Math. Soc. 132, 3387-3395.

[23] A. Spătaru. (2014). Convergence and precise asymptotics for series involving selfnormalized sums. Journal of Theoretical Probability DOI 10.1007/s10959-014-0560-1. 